
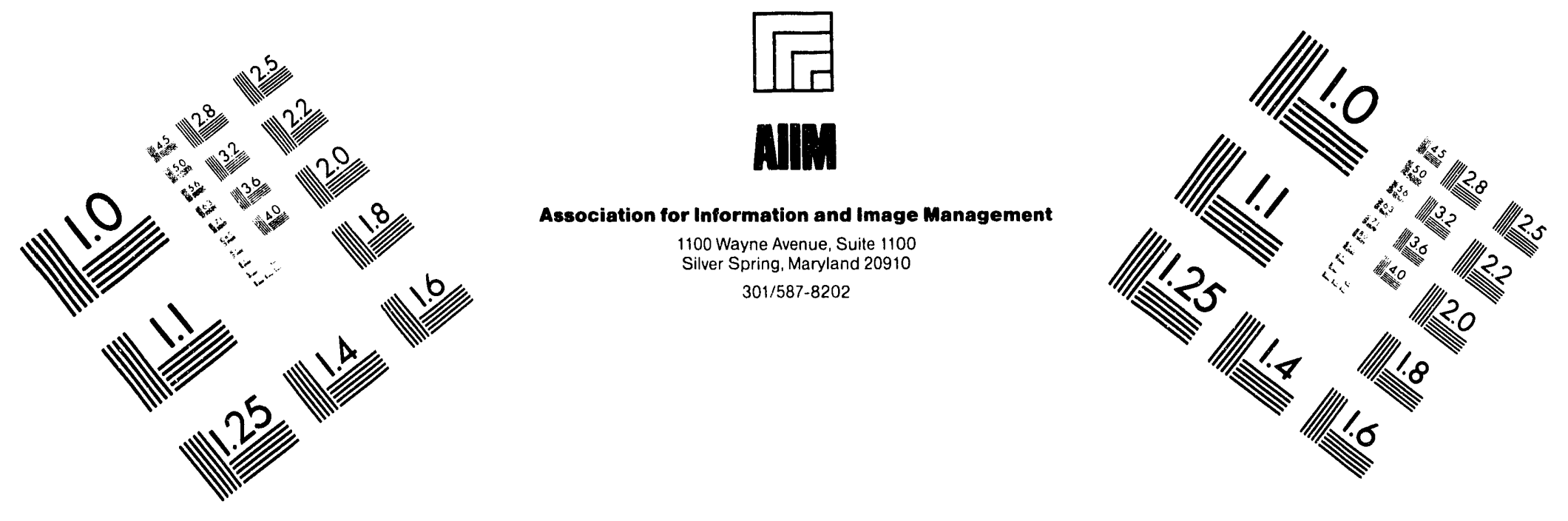

\title{
Centimeter
}

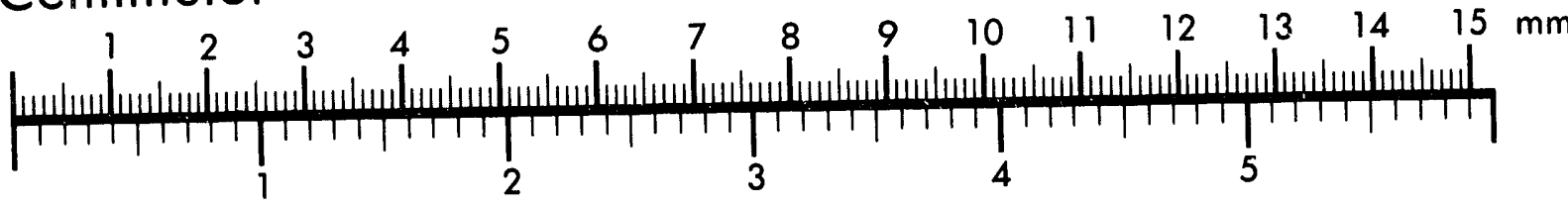
Inches
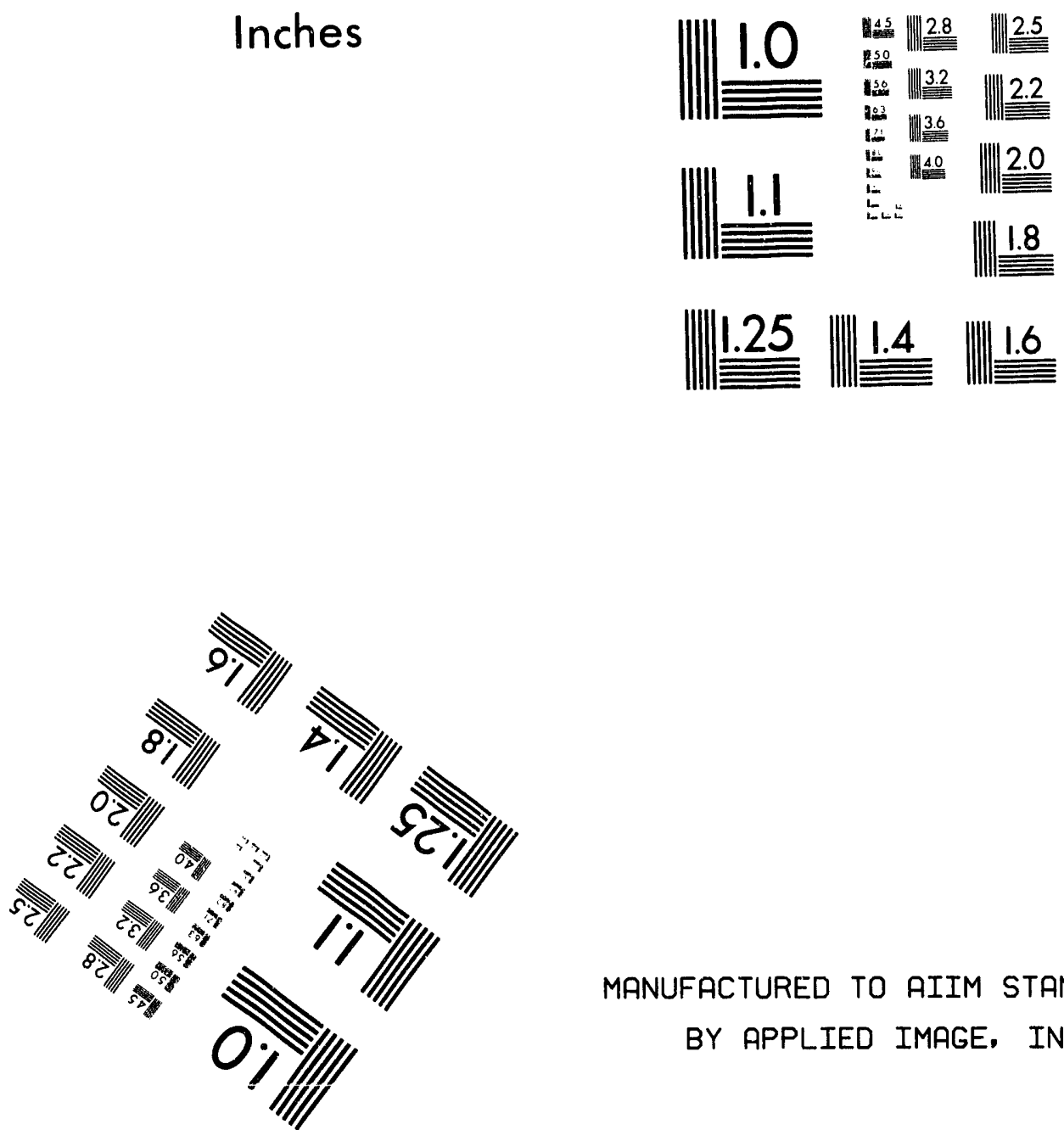

MANUFACTURED TO AIIM STANDARDS

BY APPLIED IMAGE, INC.

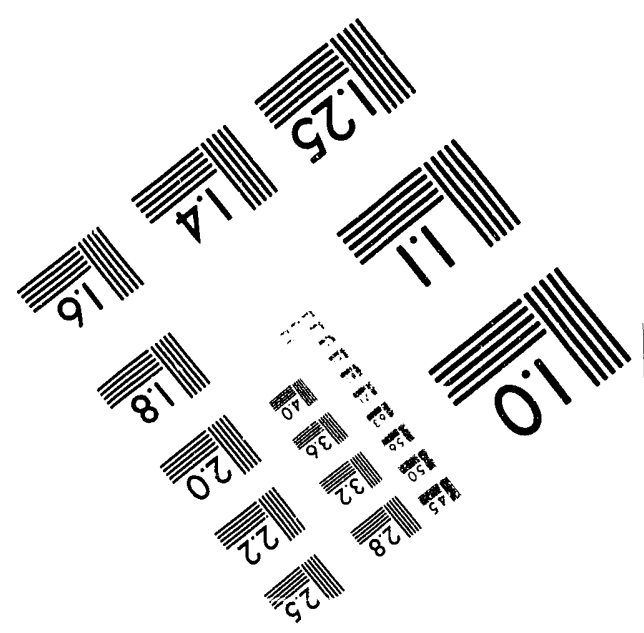



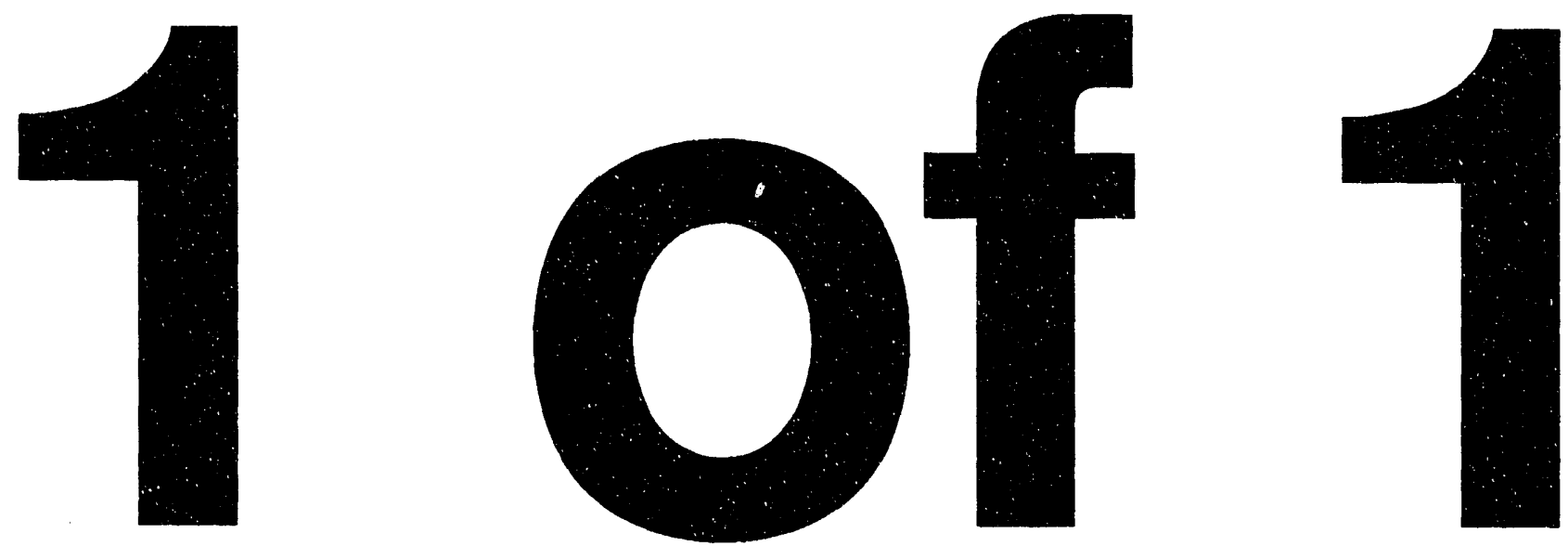


$$
\begin{aligned}
& \text { Progress Report } \\
& \text { DEE/ER/45315-17 }
\end{aligned}
$$

\section{BOUNDARY STABILITY UNDER NONEQUILIBRIUM CONDITIONS}

GRADUATE STUDENT INFORMATION

DEGREES AWARDED UNDER THIS PROGRAM

$\begin{array}{llc}\text { Ph.D } & \text { Yu Sang Ho } & 1991 \\ \text { M.S. } & \text { Blaine Danley } & 1991 \\ \text { M.S. } & \text { Thomas Lillo } & 1989 \\ \text { M.S. } & \text { Jennifer Trice } & 1989\end{array}$

Student support this year

Expected graduation date

Thomas Lillo

Jun Ho Choy

Weimen Tao

Eric McCarty

R. S. Kedia

Feb. 195; with Ph.D

Summer 1993 with Ph.D

Summer 1994 with Ph.D

Fall 1992 with M.S.

February 1993 with M.S.

PUBLICATIONS SUPPORTED BY DOE DE-FG02-87ER45315

1. S. A. Hackney, "Experimental Observation of Triple Junction Behavior During DIGM," Scripta Met., 22. 1255 (1988).

2. T. Lillo, M. R. Plichta, and S. A. Hackney, "In Situ Studies of Intrinsic Grain Boundary Structure During Thermal Cycling," Ultramicroscopy, 29, 257 (1989).

3. S. A. Hackney, "Experimental Studies on the Accommodation Dislocation Pattern for a Low Symmetry Gradient Orientation," Materials Science and Engineering, A113, 75-84 (1989).

4. S. A. Hackney and G. C. Ojard, "Grain Boundary Grooving at Finite Grain Size," Scripta Met., 22, 1731 (1988).

5. J. K. Lee and T. H. Courtney, "Two-Dimensional Finite Difference Analysis of Shape Instabilities in Plates," Met. Trans., 20A, 1385 (1989).

6. J. K. Lee and S. A. Hackney, "Coherency Strain of an Overgrown Island," Chemistry and Defects in Semiconductor Heterostructures, MRS Proceedings, Vol. 148, 315 (1989).

7. J. K. Lee, "Inclusions with and without Free Surfaces in Plane Strain," Micromechanics and Inhomogeneity, Springer-Verlag, New York, p. 213 (1989).

8. S. A. Hackney, R. Fonda, and G. J. Shiflet, "Interfacial Structure of $\Theta$ Precipitates in Al-5\%Cu Alloy," Materials Science and Engineering, A113 (1989).

9. T. Lillo, M. R. Plichta, and S. A. Hackney, "Direct Observation of Grain Boundary Dislocation Motion Due to Thermal Stresses," Scripta Met.,24,396 (1990)

10. S. A. Hackney and R. Lynch, "Experimental Observations on Epitaxial $\mathrm{NiSi}_{2}$ Islands on $<111>\mathrm{Si}$," Scripta Met., 24, 681 (1990)

11. Yu Sangho and S.A. Hackney, "On the Shape Change of a Nonoequilibrium Facetted Microcrystal", Scripta Met., 24, 2077 (1990)

12. S.A. Hackney and T. Lillo, "Grain Boundary Structural Disruption Under Thermal 
Stress", Scripta Met., 24, 1653 (1990)

13. S.A. Hackney, "On the Stability of the Rayleigh Spheroidization Process", Scripta Met., 25, 799 (1991)

14. J. K. Lee, "Computer Simulation of the Effect of Coherency Strain on Cluster Growth Kinetics," Met. Trans., 22A, 1197 (1991).

15 J. K. Lee, J. H. Choy and Y. Choi, "Equilibrium Shape and Heterogeneous Nucleation Barrier at Spherical Interfaces," Surface Science, 256, 147 (1991)

16. J. K. Lee, J. L. Trice, S. A. Hackney and M. R. Plichta, "Capillarity Induced Morphological Instabilities," Computational Materials Science, NRIM, Japan, p. 111 (1991)

17. M. H. Yoo and J. K. Lee, "Deformation Twinning in h.c.p. Metals and Alloys," Phil. Mag., 63A, 987 (1991).

18. S.A. Hackney, "On Grain Boundary Dissociation During Diffusion and Stress Induced Boundary Migration", Scripta Met., 25, 1023 (1991)

19. S.A. Hackney, "Morphological Instabilities and The Branching Mechanism at the Initiation of the Eutectoid Transformation", Scripta Met., 25, 1453 (1991)

20. S.A. Hackney and W.W. Milligan, "In Situ Deformation Studies of Ductile/Brittle Laminates", Ultramicroscopy, 37, 79 (1991)

21. T.Lillo, S.A. Hackney, and M.R. Plichta, " On the Origen of Ghost Boundaries During In Situ Strain Induced Migration" Ultramicroscopy, 37, 294 (1991)

22. J. K. Lee and M. H. Yoo, "Theory of Shape Bifurcation during Nucleation in Solids," Met. Trans., 23A, 1891 (1992).

23. J. K. Lee, J. H. Choy and Y. Choi, "The Gibbs-Wulff Construction and Critical Nucleus Morphology at a Curved Substrate or Interphase Boundary." MRS Proceedings, Vol. 230, 121 (1992)

24. J. H. Choy, S. A. Hackney and J. K. Lee, "Morphological Changes in Rod-Shaped Precipitates with Internal Boundaries: Finite Difference Analysis," in the MRS Proceedings of Interface Dynamics and Growth, in press.

25. R. M. Aiken, S. Elangoven, T.G. Zocco, M.R. Plichta, "Kinetic Equations for Concurrent Size and Shape Coarsening by the Ledge Mechanism", Met. Trans. A, 22A, 1381 (1991).

\section{SUBMITTED MANUSCRIPTS:}

S.A. Hackney, "Edge Instabilities in Thin Plates Studied By In Situ Transmission Electron Microscopy" Submitted to Ultramicroscopy

S.A. Hackney, "An Analysis of Instabilities at the Initiation of the Eutectoid Transformation Using a Kinetic Analogue" Submitted to the Journal of Mechanical Behavior of Materials.

J.H. Choy, S.A. Hackney, and J.K. Lee, "Nonlinear Stability Analysis of the Diffusional Spheroidization of Rods" Submitted to Journal of Applied Physics.

R. Kedia, T.Lillo, Q. Horn, and S.A. Hackney, "Edge Instabilities in Thin Plates with Spatial Variations in Thickness", Submitted to Scripta Met. 


\section{MANUSCRIPTS IN PREPARATION:}

Yu Sangho and S.A. Hackney, "Lamellar Instabilities in the Cellular Transformation", for Acta Met.

E.D. McCarty and S.A. Hackney, "Stability of Extrinsic Dislocations at the $\mathrm{Al} / \mathrm{SiO}_{2} \mathrm{Phase}$ Boundary", for J. Mat. Res.

M.R. Plichta, S.A. Hackney, and J.K. Lee, "Structural Stability of Gliding Tilt Boundaries", for Met. Trans.

R. Kedia and S.A. Hackney, "Observation of Stress Induced Instabilities on Single Crystal Free Surfaces", for Scripta Met.

\section{Summary and Condensed Research Proposal}

\section{Introduction}

The study of interfaces has long been justified using the idea that boundaries are often the "weak link" or determining factor in the lifetime and behavior of a material during service (or even processing). The purpose of this work is the study of the microscopic, nanometer-scale behavior of these interfaces under the nonequilibrium conditions which prevail during actual service or during materials processing. This, of course, is not an end in itself. To truly understand the microscopic phenomenon, it must be related to the overall, macroscopic, nonequilibrium conditions in terms of thermodynamic driving forces defined by (near) local equilibrium. The mission, simply put, is to study the phenomena and the physics behind macroscopic boundary instabilities in a systematic manner which allows detailed comparison between theory and experiment. The Department of Energy has put us in a position to carry out such a program by providing 1989 instrumentation funding (Project Directors: S. A. Hackney and M. R. Plichta) for a state-of-the-art, medium high voltage TEM for the purpose of in situ, dynamic studies of interfaces and 1992 instrumentation funding (Project Director: S. A. Hackney) for a molecular beam epitaxy system (MBE) dedicated to systematic and reproducible interface studies.

\section{Grain Boundaries}

\section{A. Summary of Results on Grain Boundary Stability \\ 1. Positional Stability of Grain Boundaries/Grain Boundary Dislocations under Applied Stress.}

Our group has made direct, in situ TEM observations of nonconservative [6] grain boundary dislocation motion and associated grain boundary migration on asymmetric, low angle $\left(<15^{\circ}\right)$ grain boundaries in high purity $\mathrm{Al}$ under conditions of cyclic thermal stress [3] and noncyclic thermal stress [7]. These were the first observations made on this type of boundary.

We have been able to obtain convincing evidence for self diffusion induced grain boundary migration when the grain boundary is under stress. A simultaneous grain 
boundary migration/self diffusion process is obseved. This results in an experimentally measurable thickness variation of the thin film in the migrated region. The analogy of this coupled diffusion/migration process on a grain boundary under stress with DIGM induced by second element diffusion is unmistakable. The results suggest that coupled diffusion/ migration of grain boundaries does not require alloying induced Kirdendall effects, coherently strained layers, mixing free energy changes, or grain boundary free energy changes. These experiments do not exclude these as possible driving forces for DIGM, but they do indicate that diffusional stresses of the type discussed by Balluffi and Cahn [9] and suggested by Shewmon [10] could drive the migration process. It should be noted that a quantitative hypothesis based on the experimental observations has been developed which examines the link between applied stress, GBD motion, grain boundary migration velocity, and thickness change. This hypothesis is being used as a framework for experimental design so that the quantitative comparison between theory and experiment required for refinement and testing of hypothesis is possible.

Because of the connection between grain boundary migration in a composition field and dislocation wall migration in a composition field [11], we have also studied misfit dislocation dynamics during diffusion using in situ techniques [12].

\section{Structural Stability of Grain Boundaries during DIGM and under Applied Stress}

Using in situ TEM techniques, we have studied the conservative/nonconservative motion of low angle symmetric tilt boundaries in Al under conditions of cyclic thermil Using in situ TEM techniques, we have studied the conservative/nonconservative motion of low angle symmetric tilt boundaries and more general low angle boundaries in Al under conditions of cyclic thermal stress and have produced new and unexpected results concerning the morphological and structural stability of such boundaries. These appear io be the first direct experimental observations of tilt boundary migration in fcc materials under applied stress, and certainly the first reported in situ study of conservative/ nonconservative tilt boundary migration at elevated temperatures. The structural stability, or stability of a grain boundary against dissociation under applied stress, is a problem which has never before been considered in any experimental detail, although Read and Shockley did discuss the possibility qualitatively. We have found that grain boundaries in Al can undergo dissociation and even "disintegrate" (break up into individual lattice dislocations. under conditions of applied stress (thermal stress) (Appendix 1 and 7). A connection with the phenomenon of DIGM has been made in the comparison of grain boundary behavior (dissociation) under stress with that during DIGM.

\section{B. Proposed Grain Boundary Experiments and Analysis}

\section{Experiments on Grain Boundaries Under Applied Stress}

We propose to study the effects of three variables-stress tensor, temperature, and grain boundary misorientation-on grain boundary behavior in order to test the various hypotheses concerning grain boundary behavior. For these experiments to produce results 
which can be used for comparison with theory, it is necessary to control or have sufficient knowledge of the experimental conditions. For example, it will be necessary to produce specific bicrystal thin films in a systematic and reproducible manner. The variation of the stress state and temperature are also of interest as these variables are thought to affect the behavior of the grain boundary dislocations and thus the grain boundary.

The dependent variables that must be measured in order for there to be a quantitative comparison between theory and experiment are the grain boundary velocity, the GBD velocity, and the thickness change behind the migrated grain boundary. Changes in crystal thickness during in situ straining experiments have been observed in two studies. Our study also revealed concurrent migration of the grain boundary, leaving behind a region of differing thickness. We also find that this thickness change varies for different thermal cycles. This suggests that a variation in stress state and/or temperature can affect the relative amounts of grain boundary migration and the associated diffusional creep. Our new TEM facility will allow the application of the CBED technique for local crystal thickness determination.

\section{Grain Boundary Instability in Nonhomogeneous Composition Fields: A Direct Comparison with Stress Induced Instabilities}

We propose to study instabilities in grain boundary structure and grain boundary position during binary diffusion of $\mathrm{Cu}$ into $\mathrm{Au}$ thin film bicrystals and $\mathrm{Ag}$ into $\mathrm{Au}$ thin film bicrystals by using the MBE system to bond or deposit lithographically designed islands onto the surface of MBE grown bicrystals. The bicrystal orientations would be of the same orientation as in the previous section. In situ experiments in the hot stage using our new microscope facility would be employed to study the DIGM process at high resolution. We realize that in situ experiments have been done previously, but never with the goal to compare DIGM with stress induced migration. This experiment will allow a direct comparison between boundary structure behavior for stress induced instabilities and chemical diffusion induced instabilities. Why is this important and why does it need to be done? There is now good experimental evidence (provided by Yoon and coworkers; see, for example, [16]) that the atomic size effect is indeed important and that grain boundaries are stable in the presence of chemical diffusion unless a lattice parameter change (strain) is involved. If stress is the important factor driving DIGM, then it should not matter whether a given state of stress at the grain boundary results from diffusion in an alloy or an external load in a pure material. Our own experiments show that an applied stress can result in self diffusion induced grain boundary migration in a pure material. We hope to be able to answer the question of how important stress is in the phenomenon of DIGM by comparing boundary structure/positional instabilities (grain boundary migration mechanism) during chemical diffusion with that associated with an applied stress.

In addition to the direct comparison between diffusion and stress induced grain boundary migration, we propose to experimentally model diffusional stresses at the grain boundary with an applied stress. The stress states that have been considered to exist during DIGM can be described as (1) stress axis normal to the boundary plane [10] and (2) stress axis parallel to the boundary plane but the "load" applied to only one side of the grain boundary [18] (coherency strain). Our proposed approach is to experimentally model these two stress states using the in situ TEM high temperature straining stage and to compare the nanoscale and macroscopic behavior of a grain boundary under stress with the behavior 


\section{during DIGM.}

\section{Surfaces and Thin Films}

\section{A. Summary of Results on Surface/Thin Film Instabilities}

\section{Hillock Growth on Microcrystalline and Single Crystal Surfaces}

Our approach has been to conduct concurrent experiments on microcrystalline and single crystal surfaces. The behavior is analogous except that abnormal grain growth occurs within hillocks on microcrystalline films. This is a complicating factor relative to hillock growth on single crystal surfaces as this changes (reduces) the retarding capillarity forces [23]. An important experimental result we have obtained relates to hillock growth on single crystal surfaces. Epitaxial hillocks much, much smaller than the grain size can result from applied stress. This result challenges the concept that hillock growth in thin films requires the presence of grain boundaries as sources/sinks for vacancies. To our knowledge, our work is the first controlled experimental verification that free surfaces of single crystals with tangential stresses will exhibit instabilities which will grow against curvature as predicted by theory $[19,20]$. The results do raise an important point concerning the application of theory to our experiment. We observe hillocks only. The material to form the hillocks evidently diffuses from relatively a broad expanse of the neighboring film because the change in contrast associated with any localized thinning of the film is not detectable. This asymmetry in the thickening/thinning process in the film is not predicted in first order, linear theory. We have shown how it is possible that nonlinear effects may bias the experimental observation toward hillock growth. However, the most obvious suggestion is that the hillock bias results from the compressive state of stress. Unfortunately, free surface instability theory as applied to single crystal surfaces considers strain energy terms only, which depend on the square of the stress. Thus the sign of the stress does not effect the instability form. Further experiments designed to examine the role that the sign of the stress plays in biasing the experimental observations should be carried out to examine this critical issue.

\section{Plate Edge Instabilities}

Recent publications have shown that edge instabilities of plates, films, cracks and other two dimensional structures is an area of much interest [24-27]. Using in situ TEM techniques, we have been able to study the dynamics of shape relaxation at the edges of thinned TEM foils where very high curvature terms exist. We have observed the dynamics of the development of periodically spaced cylindrical protrusions perpendicular to the plate edge. These protrusions are then observed to undergo Rayleigh spheroidization.

The spheroidization of the rods formed by the edge instability is an important step in the shape coarsening of two dimensional structures. These rods may be either single or poly crystalline in nature, depending on the nature of the structure from which they were produced. We have studied several new aspects of this old problem, specifically relating to understanding the scatter in the experimentally observed spheroidization wavelength. Eckhause instabilities have been discovered within the spheroidization process and the 
theoretical analysis provides some rationalization for the experimental observations. The new findings show that there is a stable band of wavelengths which may form during the spheroidization process and this band width is strongly dependent on the initial conditions. It is predicted that the wavelength expected from maximum growth rate considerations has only conditional stability and will only be observed when the initial conditions are of a specific type.

\section{Coherently strained islands.}

Following Eshelby's classic work on elastic inclusions, several investigations $[28,29]$ have been made on the stress field and strain energy of inclusions having such free surfaces. Because of complex boundary conditions associated with the problems, however, only a few cases have been solved and many remain unsolved. In this investigation, we have studied, by means of atomistic approach, the stress field of a free surface or an interphase boundary whose geometry is not readily amenable to a continuum mechanics analysis. Whenever they are possible, of course, we have compared the results of the atomistic approach with those of continuum elasticity. For an undergrowth whose one side is free of traction, a maximum principal stress analysis reveals stress concertrations in a tensile mode at its traction-free side, indicating a possibility of crack initiation for a brittle undergrowth. For an overgrowth whose three sides are free of traction (coherently strained surface island), a number of interesting results are found. Unlike an undergrowth or inclusion, the stress is mostly confined in the neighborhood of the overgrowth-substrate interface. For a positive misfit strain case, the stress field starts with a strong compressive state at the interface area and diminishes as the distance from the interface increases. However, nearby the substrate, the traction-free perimeter of an overgrowth is also found to be in a tensile stress mode. Quite different from the case of inclusions and undergrowths is that the total strain energy associated with an overgrowth approaches an asymptotic value as its thickness increases. That is, the strain energy density of an overgrowth is found to depend on its shape and also on its size.

\section{B. Proposed Experiments and Analyses on Surfaces/Thin Films}

\section{Epitaxial Hillock Growth and Edge Instabilities on Single Crystal Films Under Stress.}

Once again, our experiments in this area are designed to accommodate detailed comparison between theory and experiment. Hillock growth characteristics on single crystal film surfaces under stress will be compared directly to the relevant aspects of theory in [19] and [20]. In our bilayer foil geometry, it will be necessary to consider contributions from both the free surface boundary conditions developed in [19] and the variation in cross section area considered in [20]. The bilayer foils are heated in situ in the transmission electron microscope to develop the thermal stress which drives the hillock growth. Since there is a slow variation in the $\mathrm{Al}$ thickness but the constraining $\mathrm{SiO}_{2}$ layer is relatively constant, there is a slow variation in the thermal stress. To first order, the thermal stress magnitude is proportional to the thickness of the Al foil. This set up allows the study of surface behavior over a range of thermal stress magnitudes within a single wedge shaped specimen.

We believe it is important to make direct and quantitative comparisons between 
thermal stress induced surface instabilities on single crystal films and microcrystalline films. This is one way to understand the contribution that grain boundary creep and migration will make to the overall phenomenon. Using the new MBE facility, it will be possible to deposit a given material in either microcrystalline or single crystalline form on a given substrate by controlling the deposition rate and the temperature of the substrate during deposition. A similar thermal stress will develop in the two types of films if they are allowed equilibrate at the same low homologous temperature before being heated to a high homologous temperature. Using the MBE system will of course allow for such experiments to take place in ultra clean environments under highly reproducible conditions.

In the area of plate edge instabilities, the MBE system can be used to good effect in the preparation of single crystal specimens of controlled and uniform thickness for kinetic studies. There is some difficulty in comparing our experimental results with theory as there is no kinetic theory based on the general solution to the governing partial differential equations. Further theoretical work is required before full comparison with experiment is possible.

Our group also proposes to examine the effect of applied stress on the fingering instability at the edges of thin foils. This is an important aspect of this problem as two dimensional structures are often exposed to thermal or applied stresses during service. The geometry we wish to study is a single crystal foil ( $\mathrm{Au}$ or $\mathrm{Cu}$ ) with a (near) circular hole in the middle (probably produced by ion milling or electropolishing to perforation aided by lithographic masking). This will then be strained in situ in the TEM hot stage. The state of stress is then approximated as a plate with a hole pulled in tension. Stress intensification should occur nn the hole edge on the axis perpendicular to the stress axis, and the value of tangential stress varies with edge orientation with respect to the tensile axis. We wish to study the effect the radial orientation of the hole edge (with respect to the tensile axis) has on the mechanism, kinetics, and morphology of plate edge instabilities. Because the edge instability can be thought of as a form of hillock growth in two dimensions, the hole edge section parallel to the stress axis should be much more unstable with respect to instabilities than other hole edge sections with different orientations relative to the stress axis.

\section{Two-Phase Surface Instabilities in Island Growth of Thin Films}

Many coherently stressed thin films grow initially as islands. It has been pointed out in [19] that there are many physical analogies between this process and hillock growth in stressed solids of one phase. The relaxation of stress in coherently strained islands has been related to the stress relaxation in the hillocks [19]. Our theoretical results reviewed actually model the strain in a coherently stressed island and agree with the concepts outlined in [19]. Evidently, the close proximity of stress free surfaces in the island geometry cause the strain to be localized at the interface and shared between the island and the substrate. Our results suggest that as the islands become larger in diameter (thickness constant), the strain is delocalized and concentrated in the thin island. This increases the strain energy and, we believe, destabilizes the island with respect to inverse coarsening. That is, the splitting of a single island into two smaller islands may decrease the strain energy to a greater extent than the increase in capillarity energy. To examine this possibility, we propose two theoretical approaches and one set of experiments. Theoretically, the perturbation technique will be applied to both our computer results and the analytical iheory of Srolovitz. 
In both cases, we examine the stability of a partial cut in the middle of the island Experimentally, we wish to study the coarsening of coherently strained islands of $\mathrm{NiSi}_{2}$ on $\mathrm{Si}$ surfaces prior to misfit dislocation nucleation in as systematic manner as possible. We have already observed abnormal coarsening behavior in this well studied system with only two size classes present $(-1 \mu \mathrm{m}$ diameter and $\sim 0.1 \mu \mathrm{m}$ diameter $)$ [30]. The aspect which sets these proposed experiments apart from all the other studies on $\mathrm{NiSi}_{2}$ is the addition of a ternary element to systematically vary the lattice parameter of the $\mathrm{NiSi}_{2}$ phase. It has been shown [31] that $\mathrm{Al}$ will substitute for $\mathrm{Si}$ in nickel silicide and that the corresponding change in lattice parameter actually allows for an exact lattice match with the silicon substrate. Not only is this significant to our particular research problem but may also be of great technological significance. The exact lattice match between the semiconductor and metallization layer (the silicide) will allow for strain free semiconductor/metal/ semiconductor layered structures. The availability of the new MBE facility in our group makes this type of experiment possible by allowing the codeposition of $\mathrm{Ni}, \mathrm{Al}$, and $\mathrm{Si}$ onto $\mathrm{Si}$ substrates under ultraclean and controlled conditions. The anisotropic behavior of the surfaces and interfaces are well known in this system and this will add a complicating factor. It should be noted, however, that we have some experience dealing with anisotropic properties, especially as they relate to coarsening and growth.

\section{ON THE INTERRELATIONSHIP OF RESEARCH TOPICS}

The perceptive reader will have already recognized the need for study of grain boundary and surface/thin film instabilities under both the conditions of applied stress and chemical diffusion. The grain bcundaries and surfaces appear to respond to local conditions of stress. It does not appear to matter whether this stress is due to an external load or due to a composition gradient. Of significance here is that stress induced grain boundary migration and diffusion induced grain boundary migration may be the same phenomenon. This is, of course, a hypothesis which has developed from our first two years of experiments, and our proposed experiments and analysis are meant to test this idea.

Any explanation for simultaneous grain boundary/surface instability in microcrystalline thin films requires an understanding of both stress shielding within the hillock and abnormal grain growth associated with nonequilibrium conditions. This cannot be obtained by studying just surface instabilities or just grain boundary instabilities. Thus the need for concurrent study of grain boundary instability under applied stress and diffusional stress together with surface instabil 'es under applied stress and diffusional stress. In addition, the surface instability problem is closely related to the problem of coherently strained surface islands of a second phase. To understand this technologically important problem also requires the consideration of several other physical phenomena such as nonequilibrium shape effects, and strain effects on phase equilibria. This requires the study of phase boundary behavior under applied stress and coherency stress in an attempt to understand the physical phenomena associated with the growth and coarsening of coherently strained surface islands and self stressed second phase particles in general.

\section{REFERENCES}

1. H. Fukutomi and T. Kamijo, Scripta Met., 19, 195 (1985).

2. M. Kaspar and H. Gieiter, Acta Met., 32, 1903 (1984). 
3. T. Lillo, M. R. Plichta, and S. A. Hackney, Ultramicroscopy, 29, 257

(1989).

4. S. A. Hackney, F. S. Biancaniello, D. N. Yoon, and C. A. Handwerker, Scripta Met., 20, 937 (1986).

5. S. A. Hackney, Scripta Met., 21, 371 (1987).

6. R. W. Balluffi, Grain Boundary Structure and Kinetics, ed. R. W. Balluffi, p. 297, ASM, Metals Park, OH (1980).

7. T. Lillo, M. R. Plichta, and S. A. Hackney, accepted to Scripta Met.

8. S. E. Babcock and R. W. Balluffi, Acta Met., 37, 2357 (1989).

9. R. W. Balluffi and J. W. Cahn, Acta Met., 29, 493 (1981).

10. P. G. Shewmon, Acta Met., 29, 1567 (1981).

11. Y.J.M. Brechet, J: R. Dryden, and G. R. Purdy, Acta Met., 37, 2261 (1989).

12. S. A. Hackney, Materials Science and Engineering, A 113, 75 (1989).

13. S. A. Hackney, Scripta Met., 22, 1255 (1988).

14. Longworth, H.P., Doctoral Thesis, MIT, Feb. 1992, p. 233

15. R.C. Ecob, Scripta Met., 20,1001 (1986)

16. H. K. Kang, S. A. Hackney, and D. N. Yoon, Acta Met., 36, 695 (1988).

17. R.T.P. Whipple, Phil. Mag., 45, 1225 (1984).

18. M. Hillert, Scripta Met., 17, 237 (1983).

19. D. J. Srolovitz, Acta Met., 37, 621 (1989).

20. F. C. Larche and J. W. Cahn, J. of Research NBS, 89, 467 (1984).

21. W. B. Pennabaker, JAP, 40, 394 (1968).

22. W. D. Nix, Met. Trans. A, 20A, 2217 (1989).

23. S. A. Hackney, Scripta Met., 22, 1273 (1988).

24. T. H. Courtney and J. C. Malzahn Kampe, Acta Met., 37, 1747 (1989).

25. E. Jiran and C. V. Thompson, J of Electronic Materials, vol. 19 (1990) 1153

26. F. F. Lange and D. R. Clarke, J. American Ceramic Soc, vol. 65 (1982) 502

27. J. Rodel and A. M. Glaeser, J. American Ceramic Soc, vol. 73 (1990) 592

28. Y. P. Chiu, J. Appl. Mech., 45, 302 (1978).

29. E. Tsuchida and T. Mura, J. Appl. Mech., 50, 807 (1983). 
30. S. A. Hackney and R. Lynch, Scripta Met.

31. A. Wittmann, K.O. Burger und H. Nowotny, Mh. Chem., Bd. 93 (1962) 674.

\section{DISCLAIMER}

This report was prepared as an account of work sponsored by an agency of the United States Government. Neither the United States Government nor any agency thereof, nor any of their employees, makes any warranty, express or implied, or assumes any legal liability or responsibility for the accuracy, completeness, or usefulness of any information, apparatus, product, or process disclosed, or represents that its use would not infringe privately owned rights. Reference herein to any specific commercial product, process, or service by trade name, trademark, manufacturer, or otherwise does not necessarily constitute or imply its endorsement, recommendation, or favoring by the United States Government or any agency thereof. The views and opinions of authors expressed herein do not necessarily state or reflect those of the United States Government or any agency thereof. 

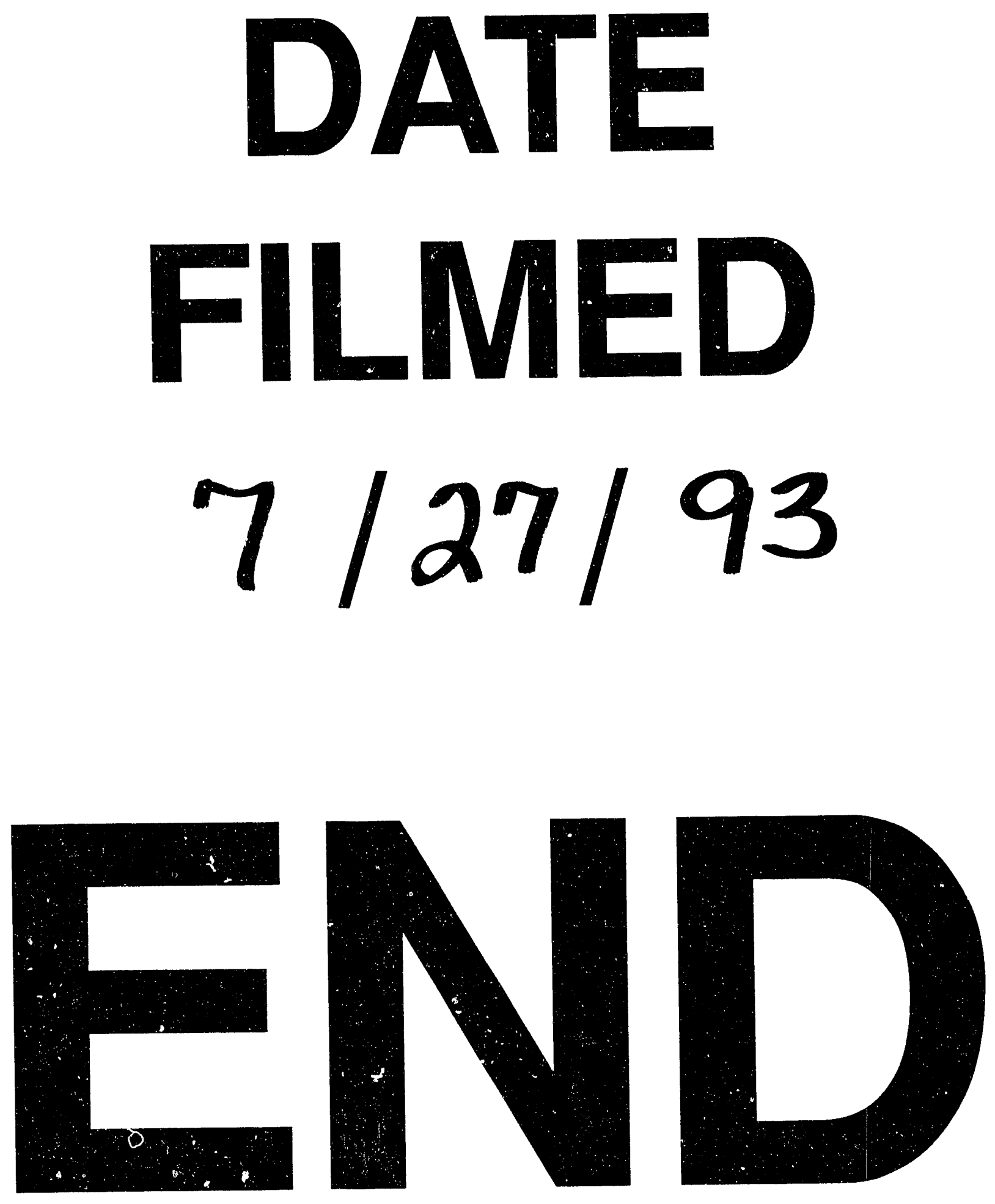
